\title{
Assessment of quality assurance program of HIV testing in Ethiopia
}

\author{
Belayneh Regasa \\ Medical Microbiology Department, Arba Minch University, Arba Minch, Ethiopia \\ Email address: \\ belayjanimen@gmail.com
}

\section{To cite this article:}

Belayneh Regasa. Assessment of Quality Assurance Program of HIV Testing in Ethiopia. American Journal of Internal Medicine. Vol. 2, No. 6, 2014, pp. 106-108. doi: 10.11648/j.ajim.20140206.13

\begin{abstract}
Background: HIV/AIDS is one of the most challenging health crises facing the world today. The availability of excellent HIV (Human immunodeficiency virus) tests does not automatically guarantee reliable result. Many steps are involved between specimen collections to the moment when reported to physician and at each step something may go wrong. Measures to control the quality of result in HIV diagnostic laboratories are extremely important, because of the consequence of either false positive or false negative results are huge. Methods: A cross sectional study conducted to assess quality assurance program of HIV testing in Addis Ababa Hospitals and Clinics, Ethiopia from May to October 2012. A well designed and structured questionnaire, Checklist and onsite observation were used to collect data. Data was processed and analyzed with SPSS version16.0. Results: Out of 20 assessed hospitals and clinics cases, 3(14\%) laboratory personnel's who conduct HIV testing were found to have no training in HIV testing. Some laboratories $2(10 \%)$ do not follow HIV testing algorithm and also $2(10 \%)$ laboratory personnel's do not know what to do in case of indeterminate result. HIV testing methods used were Rapid/ simple $20(100 \%)$, ELISA $13(65 \%)$, and Western blot $1(5 \%)$. All laboratories use controls that are supplied with kit but $2(10 \%)$ laboratories use external control (pooled sera) additionally. Seventeen (85\%) uses manual (guidelines) supplied with kits but none of them uses SOPs (Standard operating procedures). There was poor participation in EQA (External Quality Assessment) program (50\%). Conclusion: This study showed that there is lack of qualified human resources, not following HIV testing algorithm and poor participation in External quality assessment program. Therefore having a good quality assurance program and participation in external quality assessment scheme is indispensable. In addition, provision of refreshment training for laboratory personnel's' who conduct HIV testing and involving them in planning and management will increase the quality of HIV testing.
\end{abstract}

Keywords: $H I V, H I V$ Testing and Quality Assurance

\section{Introduction}

HIV/AIDS is associated with high mortality and it is not only the impact on the health of the individuals, but also devastates families, communities and the political stability of the countries, especially in the poorer regions of the world. It threatens development, social cohesion, food security and life expectancy, imposing a devastating economic burden that needs urgent attention. (1,2,3). HIV(Human immunodeficiency virus) testing helps to document the existing level of the HIV epidemic in various populations, so that policy makers would be aware of the importance of the epidemic and also helps the patients know their status and prevent progression of the disease by taking antiretro viral treatments (ART) $(4,5,6)$. A good quality assurance program in HIV testing is indispensable to assure reliable result which is dependent on educational background, certification, and training of laboratory personnel, the condition of the specimens, the controls used with the test runs, the interpretation of the results and the reporting of the result (7-11).

\section{Methods and Materials}

During the period May to October 2012 a total of 20 hospitals and clinics found in Addis Ababa, Ethiopia were participated in this study. A well designed questionnaire, checklist and on site observation was made during the specified time to asses qualification of laboratory personnel's involved in HIV testing, HIV testing methods used, SOPs 
(standard operating procedures) utilization, internal and external quality control utilization. Data were entered and analyzed using SPSS version 16.0 computer software.

\section{Result}

A total of 20 governmental and nongovernmental hospitals and clinics (9 hospitals and 11 clinics) laboratories found in Addis Ababa, Ethiopia was participated in this study (Table $1)$.

Table 1. Distribution of Laboratory personnel's involved in HIV testing by sex and age $(n=20)$

\begin{tabular}{ll}
\hline Variables & Frequency (percent) \\
\hline Gender & \\
Male & $17(85)$ \\
Female & $3(15)$ \\
Age & \\
$20-25$ & $4(20)$ \\
$26-45$ & $16(80)$ \\
\hline
\end{tabular}

Among 20 laboratory personnel's 19 (95\%) were diploma holders (laboratory technicians), and 1 (5\%) was B.Sc degree holder (laboratory technologist). Among these 17 (85\%) were taken training on HIV testing but the rest 3 (15\%) did not take any training on HIV testing. Most trainees took training for 15 days once (Table 2).

Table 2. Educational level of Laboratory personnel's involved in HIV testing

\begin{tabular}{ll}
\hline Educational level & Frequency (percent) \\
\hline Technician/ diploma & $19(95)$ \\
Technologist/ B.Sc degree & $1(5)$ \\
\hline
\end{tabular}

Some Laboratories $2(10 \%)$ do not follow $H I V$ testing algorithm but the common HIV testing methods used were Rapid/ simple 20(100\%), ELISA 13 (65\%), and Western blot 1(5\%) (Table 3 and 4). Some HIV testing laboratories 2 (10\%) do not what to do in case of indeterminate result.

Table 3. Test methods used in HIV testing hospital and clinics in Addis Ababa, Ethiopia

\begin{tabular}{ll}
\hline Test methods & Frequency \\
\hline Rapid/ Simple & 20 \\
ELISA & 13 \\
Western blot & 1 \\
\hline
\end{tabular}

Table 4. Test Algorithm used in HIV testing hospital and clinics in Addis Ababa, Ethiopia

\begin{tabular}{ll}
\hline Test methods & Frequency (percent) \\
\hline Rapid/ Rapid + Rapid/ELISA & $8(40)$ \\
Rapid/ Rapid & $5(25)$ \\
Rapid/ ELISA & $3(15)$ \\
ELISA/ELISA + Rapid/Rapid & $1(5)$ \\
ELISA/ Western blot + Rapid/Rapid + & $1(5)$ \\
ELISA/ELISA & \\
Use of only one kit (no confirmation) or not & $2(10)$ \\
following HIV test algorithm & \\
\hline
\end{tabular}

All laboratories use controls that are supplied with kit but 2
(10\%) laboratories use external control (pooled sera) additionally. Seventeen (85\%) uses manual (guidelines) supplied with kits but none of them uses SOPs (Standard operating procedures). Some laboratories 6 (30\%) do not preserve border line samples for follow up. In all assessed (supervised) laboratories the result and test methods were recorded in the laboratory record books. Among those hospitals and clinics that use ELISA kit to test HIV, the statistical methods such as standard deviation, mean and coefficient of variation were used only in $1(5 \%)$ laboratory to establish acceptance value for internal quality control, external control and cut off value.

There was poor participation in EQA (External Quality Assessment) program (50\%) (Table 5).

Table 5. Participation in external quality assessment (EQA) program in HIV testing hospital and clinics in Addis Ababa, Ethiopia

\begin{tabular}{ll}
\hline Participation in EQA & Frequency (percent) \\
\hline Regular participant & $8(40)$ \\
Irregular participant & $2(10)$ \\
None participant & $10(50)$ \\
\hline
\end{tabular}

Out of 20 assessed HIV testing hospital and clinics only 10 $(50 \%)$ laboratories monitor quality control/ quality assurance program, 3 (15\%) laboratories evaluate laboratory personnel's that screen HIV and 3 (15\%) laboratories had implemented continuing education program for their laboratory personnel's who perform HIV testing.

\section{Discussion}

The importance of assuring quality of HIV testing has been identified as a key step towards ensuring reliable result and has a core role in prevention and control HIV/AIDS, which was the main purpose of this study.

In this study even though the percentage of trained laboratory personnel in HIV testing hospitals and clinics was high 17 (85\%), high turnover of trained human resource from governmental to private laboratories in general and in HIV testing laboratories in particular was still a problem, this could be partially solved by organizing continuing training programs, including HIV screening methods and quality assurance in the regular curriculum for laboratory personnel's and creating conducive environment by providing incentives to the professionals in the field(9).

WHO recommended that either Rapid/Rapid or ELISA/ Rapid or ELISA/ELISA or ELISA/Westernblot test combination can be used to confirm result in HIV screening laboratories $(12,13)$. In this study $8(40 \%)$ laboratories use Rapid/ Rapid + Rapid/ELISA, 5 (25\%) uses Rapid/ Rapid, 3 (15\%) uses Rapid/ ELISA, 1 (5\%) uses ELISA/ELISA + Rapid/Rapid, 1 (5\%) uses ELISA/ Western blot + Rapid/Rapid + ELISA/ELISA and 2 (10) laboratories uses of only one kit (no confirmation) or not following HIV test algorithm. This indicates less availability of test kits. Hence to improve the situation either the existing referral system should be strengthen or at least two rapid assays, as recommended by 
WHO and different studies should be in place (11). According to study conducted in Ethiopia, out of 42 assessed laboratories $82.5 \%$ use ELISA/Rapid and $13.3 \%$ use Rapid/Rapid (9), in contrast in this study use of Rapid/ Rapid is high. This may be due to the very easiness of the test to be performed, rapid diagnosis and rapid tests can be handled with limited training.

All laboratories in hospitals and clinics involved in this study uses controls supplied with the test kits, but some 2 $(10 \%)$ use well characterized pooled sera additionally. The short-term objectives of this exercise (using pooled sera) is to monitor the external control during each run to determine if the controls yield results that are in the range expected, and that their performance is acceptable. Once these controls and their ranges are well established, the long term goal will be use of these controls in conjunction with regular kit controls to more thoroughly continually monitor HIV test kit performance (7).

In this study none of assessed hospitals and clinics use standard operating procedures (SOPs), but study conducted in South East Asia indicates only 1 out of 13 assessed laboratories did not have SOPs (10). This low utilization of SOPs is may be due to lack of training on importance of SOPs and negligence by laboratory personnel's.

This study indicated that $10(50 \%)$ hospitals and clinics did not participate in external quality assessment (EQA) in contrast to study conducted in South East Asia in which only $15.6 \%$ did not participate in EQA (10). This may be due to lack of initiative or refreshment training for those laboratory personnel's to participate in EQA. The figure in this study is more or less the same to study conducted in Ethiopia earlier (9), hence no significant improvement made in the area till now.

Low opportunity for continuing education program was observed in this study $3(15 \%)$, which could attribute to the fact that there is scarcity of program for continuing education or updating laboratory personnel's. Periodical refreshment courses should be given to laboratory personnel's to update them with new developments.

In this study only $10(50 \%)$ hospitals and clinics monitor quality control program. This may be due to less attention given to the quality assurance of HIV test. Even though evaluation of laboratory personnel's is important for their efficacy, only $3(15 \%)$ hospitals and clinics implement this. Individual meeting should be held with each laboratory personnel's who are performing HIV testing on quality of work.

\section{Conclusion}

This study showed that there is lack of qualified human resources, not following HIV testing algorithm and poor participation in External quality assessment program. Therefore having a good quality assurance program and participation in external quality assessment scheme is indispensable. In addition, provision of refreshment training for laboratory personnel's' who conduct HIV testing and involving them in planning and management will increase the quality of HIV testing.

\section{Acknowledgements}

The author would like to thank those who were involved in this research.

\section{References}

[1] Cock KM, Marum E. A sero status- based approach to HIV/AIDS prevention and care in Africa. Lancet. 2003; 362:1847-1848.

[2] Sabati K. HIV/AIDS in Ethiopia: past, present and future. Horn Africa journal of AIDS, 2004; I (II): 70-71.

[3] Peter B, Whit tall T, Babaahmady K, Vaughan R, Lehner T, et al Effect of heterosexual intercourse on mucosal alloimmunization and resistance to HIV-1 infection. Lancet. 2004; $362: 518$.

[4] Fontanet A, Woldemichael T. The Ethiopia Netherlands AIDS research project (ENARP): description and major findings after 4 years of activities, Ethiop Med J. 1999; 37(1);19.

[5] Van Dyck E, Meheus AZ, Piot P, et al. Laboratory diagnosis of sexually transmitted diseases, WHO, Geneva, 1999:86-95.

[6] Goldsby R, Kindt TJ, Osborne BA, Kuby J, et al. Immunology, $5^{\text {th }}$ ed. New york, W.H.Freeman and company, 2003

[7] Contantine N, Collahan JD, Watts DM, et al. Retroviral testing: essential for quality control and lab diagnosis, $2^{\text {nd }}$ ed. CRC press, inc. bola Raton, USA, 1992

[8] WHO/UNAIDS, Guide lines for organizing national external quality assessment scheme for HIV serological testing 1996:1-5

[9] Tegbaru B, Melese H, Tamene W, et al. The status of HIV screening Laboratories in Ethiopia: achievement, problems encountered and possible solutions. Ethiop J Health develop. 2002;16 (2):209-215

[10] Quality Assurance in HIV testing, blood safety and clinical Technology, Pune, India, 2003:24-28.

Http://www.whosea.org

[11] Meles H. Tegbaru B, Messele T, et al. Evaluation of rapid assays for screening and confirming HIV-1 infection in Ethiopia. Ethiop Med J. 2002, 40:27

[12] WHO, operational characteristics of commercially available assays to determine Antibodies to HIV-1 and/ or HIV-2 in human sera, Geneva, January 1998 (Report 9/10. WHO/98.1)

[13] World Health organization and United Nation joint program on HIV/AIDS. Revised recommendations for selection and use of HIV antibody tests, WHO weekly Epidemiology, 1997 (12);81-88 